BULGARIAN ACADEMY OF SCIENCES

CYBERNETICS AND INFORMATION TECHNOLOGIES • Volume 15, No 2

Sofia $\bullet 2015$

Print ISSN: 1311-9702; Online ISSN: 1314-4081

DOI: 10.1515/cait-2015-0037

\title{
An Improved Path Planning Method Based on Artificial Potential Field for a Mobile Robot
}

\author{
Wenbai Chen, Xibao Wu, Yang Lu \\ School of Automation, Beijing Information Science and Technology University, 100192, Beijing, \\ China \\ Email: chenwb03@126.com
}

\begin{abstract}
To solve the problem of local minima and unreachable destination of the traditional artificial potential field method in mobile robot path planning, chaos optimization is introduced to improve the artificial potential field method. The potential field function was adopted as a target function of chaos optimization, and a kind of "two-stage" chaos optimization was used. The corresponding movement step and direction of the robot were achieved by chaos search. Comparison of the improved method proposed in this paper and the traditional artificial potential field method is performed by simulation. The simulation results show that the improved method gets rid of the drawbacks, such as local minima and unreachable goal. Furthermore, the improved method is also verified by building up a physical platform based on "Future Star" robot. The success of the physical experiment indicates that the improved algorithm is feasible and efficient for mobile robot path planning.
\end{abstract}

Keywords: Artificial potential field method, chaos optimization, path planning, mobile robot.

\section{Introduction}

Given in a complicated task environment, the robot's first task is to avoid obstacles and reach the destination as efficiently as possible. Typically, path planning can be described as the procedure in which the robot finds out the optimal collision-free path from a specified position to the destination according to certain criteria, such as minimum distance, minimum time and maximum safety. Path planning has been studied and applied in many research fields [1-3]. 
The artificial potential field method proposed by Andrews, Hogan and Khatib [4] has gained increased popularity in the field of mobile robot path planning [5-7]. In these approaches, the target exerts an imaginary attractive force on the robot, while the obstacles apply repulsive forces to the robot. The total resultant force determines the subsequent direction and speed of travel. With the characteristic of simplicity, the traditional potential field method can be implemented conveniently due to its high efficiency.

However, inappropriate definitions of the potential field equations will produce local minima of potential fields. As a result, the robot might be trapped into local situations. For example, it oscillates in the presence of obstacles and swings in narrow passages $[6,7]$. Thus, the artificial potential field method requires to be associated with some other artificial intelligence optimization algorithms, such as genetic algorithms, fuzzy and artificial neural networks, etc. [8-10].

To solve the problems above described, this paper introduces chaos optimization into path planning algorithm to improve the artificial potential field method. This paper is organized as follows. In Section 2 the algorithm principle and the problems of the potential field methods are analyzed. Section 3 presents the improved artificial potential field method based on chaos optimization. In Section 4 comparison and analysis of the improved method proposed in this paper and the traditional artificial potential field method are performed by simulation. Furthermore, the improved method is also verified by building up a physical platform based on "Future Star" robot in Section 5.

\section{Traditional artificial potential field method}

The artificial potential field method introduces field concepts of physics theory into environment planning expressions $[4,6]$. The obstacles produce a repulsive force while the goals produce an attractive force on the robot. The resultant force determined by the repulsive and attractive forces controls the moving direction of the robot. Substantially, this method is to define the virtual potential field among the moving environments of the robot. The defined potential field is the superposition of the goal's attractive field and the obstacle's repulsive field $[4,11]$.

The artificial potential field can be written as

$$
\mathrm{U}_{\text {sum }}=U_{\text {att }}+U_{\text {rep }} \text {, }
$$

where $U_{\text {att }}$ and $U_{\text {rep }}$ are the attractive and repulsive fields in which the robot locates.

As shown in Fig. 1, the total force applied to the robot is the resultant of the attractive force $F_{\text {att }}$ towards the goal and the repulsive force $F_{\text {rep }}$ from the obstacle.

$$
F_{\text {total }}=F_{\text {att }}+F_{\text {rep }} \text {. }
$$

Obviously, the resultant force $F_{\text {total }}$ determines the speed and direction of the robot. 


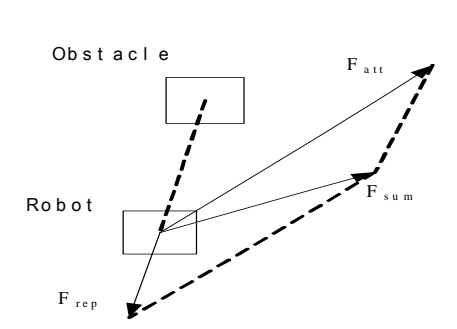

Go a I

Fig. 1. Resultant force applied to the robot

2.1. Definition of the attractive potential field

Assuming that the robot locates at coordinate $X=(x, y)^{\mathrm{T}}$ in a planar space and the coordinate of the goal point is $X_{\mathrm{g}}=\left(x_{\mathrm{g}}, y_{\mathrm{g}}\right)^{\mathrm{T}}$, the attractive potential field function can be defined as

$$
U_{\text {att }}=\frac{1}{2} k\left(X-X_{\mathrm{g}}\right)^{2},
$$

where $U_{\text {att }}$ denotes the attractive field function where the robot is located; $k$ is an attractive constant which is always greater than zero; $x$ is the coordinate vector of the robot, while $x_{\mathrm{g}}$ is the coordinate vector of the goal.

Obviously, the attractive force $F_{\text {att }}$ is a negative gradient function of the attractive field.

$$
F_{\text {att }}=-\operatorname{grad}\left(U_{\mathrm{att}}\right)=k\left(X_{\mathrm{g}}-X\right) .
$$

2.2. Definition of the repulsive potential field

Usually, the artificial repulsive potential function can be written as

$$
U_{\text {rep }}= \begin{cases}\frac{1}{2} \eta\left(\frac{1}{\rho}-\frac{1}{\rho_{\mathrm{o}}}\right)^{2}, & \rho \leq \rho_{\mathrm{0}}, \\ 0, & \rho>\rho_{\mathrm{0}},\end{cases}
$$

where $U_{\text {rep }}$ is the repulsive field of the position where the robot is located; $\eta$ is a positive scaling factor; $\rho$ is the distance between the robot and the obstacles in planar spatial coordinates; $\rho_{0}$ is the maximum distance of the repulsive field influenced by the obstacle. The obstacle has no influence on the robot's movement, if it is further away than $\rho_{0}$.

The repulsive force can be written as

$$
F_{\text {rep }}=-\operatorname{grad}\left(U_{\text {rep }}\right)=\left\{\begin{array}{c}
\eta\left(\frac{1}{\rho}-\frac{1}{\rho_{\mathrm{o}}}\right) \frac{1}{\rho^{2}} \frac{\partial \rho}{\partial X}, \quad \rho \leq \rho_{\mathrm{o}}, \\
0, \quad \rho>\rho_{\mathrm{o}} .
\end{array}\right.
$$




\section{Improved artificial potential field method based on chaos optimization}

\subsection{Principle of chaos optimization}

An early proponent of chaos theory was Henri Poincaré. Chaotic systems are predictable for a while and then appear to become random [12].

Based on the chaos theory, the chaos optimization algorithm can directly search the extremum point in a certain space $\left[a_{i}, b_{i}\right]$ by a chaotic variable. Many optimization problems can be formulated as follows:

$$
\text { Min } f(X), X=\left[x_{1}, x_{2}, \cdots, x_{n}\right] \text { s.t. } x_{i} \in\left[a_{i}, b_{i}\right], i=1,2, \ldots, n \text {, }
$$

where $f$ is the objective function, and $X$ is the decision vector consisting of $n$ variables.

According to the pattern of chaotic behavior, the search process can jump out of the local minimum easily. Moreover, the search efficiency is high.

The well-known logistic mapping is usually used to describe the chaotic motion ergodic characteristics $[8,13]$ :

$$
x_{k+1}=f\left(\mu, x_{k}\right)=\mu x_{k}\left(1-x_{k}\right),
$$

where $x_{k}$ denotes the chaotic variable and its range is $0 \leq x_{k} \leq 1$. The variable range of the control parameter $\mu$, which can control the system state, is $0 \leq \mu \leq 4$. It is easy to find that (8) is a deterministic system without any random disturbances. It seems that its long-term behavior can be predicted. But this is not true. When $\mu>3.57$ the system (8) begins to behave chaotically in an unpredictable pattern. Fig. 2 shows the logistic mapping orbit at different values of $\mu$ in the iterative trajectory.
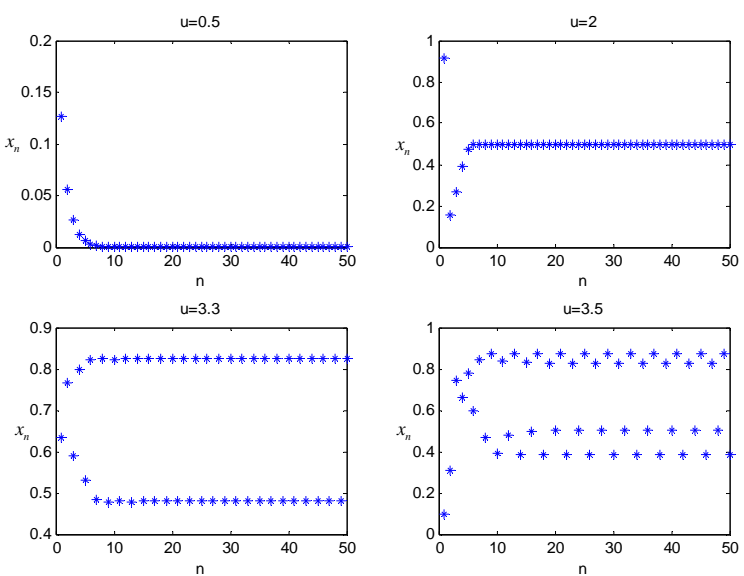

Fig. 2. Logistic mapping orbit at different $\mu$ values

When $\mu=4$ it is entirely a state of chaos. The track of the chaotic variable can traverse the whole space of interest. Fig. 3 shows the value of $x_{k}$ in an iterative trajectory, in which the initial value $x_{0}$ is 0.007 . 


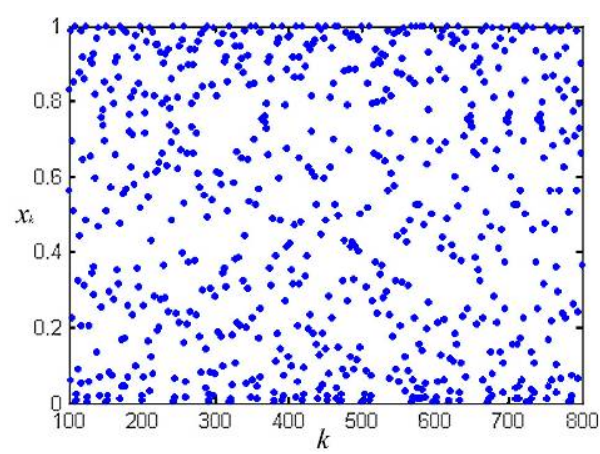

Fig. 3. Iterative sequence of the logistic map

\subsection{Characteristics of chaos optimization}

The traditional optimization algorithms, such as the descent algorithm, conjugate direction algorithm and the variable metric algorithm are all classified in deterministic optimization algorithms, which can be used to solve the convex optimization problem of the extreme point's seeking. However, the actual optimization problems are often quite complex. For example, the objective function has multiple extreme points, does not meet the requirement of convexity, is not continuously differentiable and even cannot have a specific expression. So, the traditional methods often have difficulty in solving these optimization problems.

The chaos search method is a derivative-free optimization method. It overcomes the difficulties of the traditional derivative-based optimization methods which heavily depend on the gradient information.

\subsection{Artificial potential field method based on chaos optimization}

The chaos artificial potential field method combines the chaos optimization algorithm and the traditional artificial potential field method. The potential field function is adopted as a target function of chaos optimization. The parameters needed to optimize are the step length and the potential field angle which is the moving direction of the robot. $[14,15]$

The attractive force potential function is given by the most common form

$$
U_{\text {att }}=k \rho_{\text {target }}^{2},
$$

$\rho_{\text {target }}$ is the distance between the robot and the goal; $k$ is a positive constant determined by the shape of the obstacle, which can be defined as a gravity gain coefficient. The next expression is the improved repulsive force potential of the $i$-th obstacle.

$$
U_{\text {rep } i}=\left\{\begin{array}{cc}
\lambda_{i}\left(\frac{1}{\rho_{i}}-\frac{1}{\rho_{0}}\right)^{2} \rho_{\text {tagat }}, & \rho_{i} \leq \rho_{0}, \\
0, & \rho_{\mathrm{i}}>\rho_{0},
\end{array}\right.
$$


where $\rho_{i}$ is the distance between the robot and the $i$-th obstacle; $\rho_{\mathrm{O}}$ stands for the safe distance; $\lambda_{i}$ is a positive constant determined by the shape of the obstacle as well. Thus, the total repulsive potential field can be written as

$$
U_{\text {rep }}=\sum_{i=1}^{n} U_{\text {rep } i} \text {, }
$$

where $n$ is the amount of the obstacles, including the static and moving obstacles in the working environment.

Therefore, the obtained potential field function can be adopted as a target function of chaos optimization. The optimal step length and the potential field angle of the robot can be calculated by the chaos optimization algorithm in real time.

A kind of a "two-stage" algorithm [16] is used to resolve the problem of multivariable and large solution space. The algorithm diagram of the two-stage chaos optimization is shown in Fig. 4.

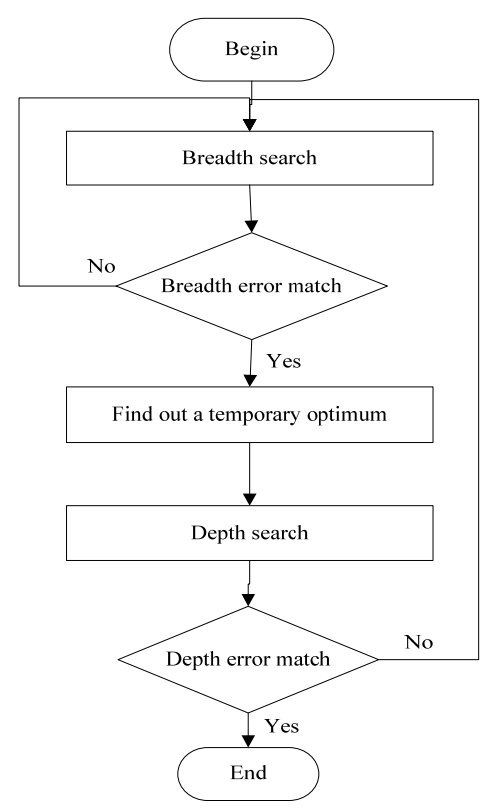

Fig. 4. Algorithm diagram of two-stage chaos optimization

The main idea of the algorithm divides the whole search space into a breadth space and a depth space. The mission of the breadth search is to find out a temporary optimum in a short time. After that, the depth search looks for the small space around the temporary optimum. During this procedure, if the algorithm is trapped at a local optimum, it goes back to the first stage. Both procedures are iterated until catching the global optimum. In the logistic iteration, with control parameter $\mu=4$, the logistic mapping turns out to be a full mapping within the interval $[0,1]$.

Because of the characteristics of the chaos optimization algorithm above mentioned, the planned path using this method can get rid of the local minima. On 
the other hand, the robot can find the path between two obstacles, which are close to each other and the two obstacles must have sufficient distance to make the robot pass.

In the path planning process above described, once the value of the potential function $U$ is relatively small, a smoothing factor should be considered. We can strengthen the attractive potential function and weaken the repulsive potential in the target function relatively according to the actual problem [17].

\section{Simulation experiment}

To verify whether this method is efficient, a simulating experiment was conducted to compare the effects of the traditional and improved artificial potential field methods under local stable situations. Assuming that the environment is partly known, the size of the robot is ignored, the initial position and the goal position of the robot are $[0,0]$ and $[10,10]$ respectively. Moreover, the obstacle is defined as a circle whose center coordinate is the center of the mass to produce the repulsive force. In this experiment the other parameters $\rho_{0}=2, k=1, \lambda_{i}=1000,0 \leq l_{j} \leq 2$, $0 \leq \theta \leq \pi .{ }_{j}$ and $\theta$ must be provided by each calculation, representing the step length and the moving directional angle of the robot.

In Fig. 5 there are two obstacles located on [4.2, 5.8] and [5.2, 4.8]. In this situation, if the traditional artificial potential field method is used, the robot may oscillate or stop at the gap of the two obstacles. Thus the robot is not able to reach the destination. Fig. 5a indicates that the robot bypasses the two obstacles, approaching the obstacles, when using the traditional artificial potential field method. However, the robot reaches the destination. In Fig. 5b, if using the chaos artificial potential field method, the robot passes the narrow path between the two obstacles although there is a local minimum of the artificial potential field method.

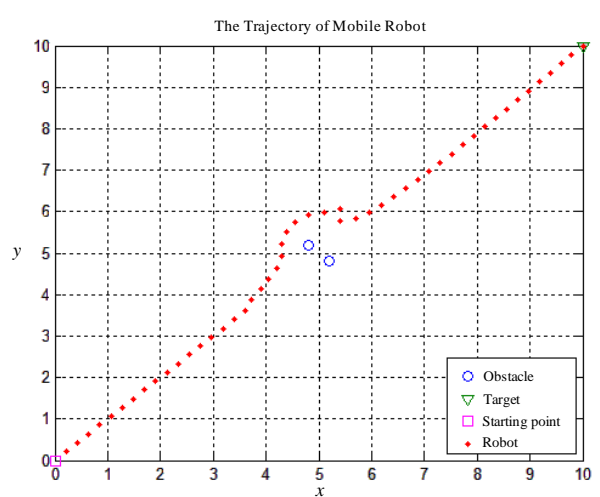

(a)

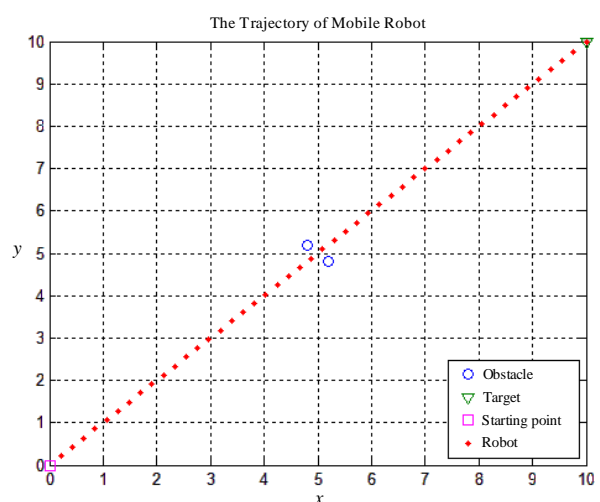

(b)

Fig. 5. Simulation comparison of the condition of trap situation due to a local minimum: Traditional artificial potential field method (a); improved artificial potential field methods (b) 
In Fig. 6 the obstacle and the goal are close to each other, their coordinates being $[9.5,10]$ and $[10,10]$ respectively. In this situation, if we use the traditional artificial potential field method, the robot may not be able to reach the goal, because the attractive and repulsive forces are counter posed with respect to each other. Fig. 6a shows that the robot moves forwards from the first step to the 40th step at a directional angle of 45 degrees due to the attractive force.

However, when the robot approaches the goal and comes into the affected range of the obstacle, the robot changes its direction suddenly. It is not able to reach the goal correctly. According to the analysis of the repulsive and attractive field models, it is the repulsive force that balances out the attractive force and the resultant force guides the robot away from the goal. In other words, the goal is not the minimum potential field position among the whole force field at present. Thus, the robot goes to the wrong direction and fails to reach the destination.

Fig. $6 \mathrm{~b}$ shows that the robot bypasses the obstacle and reaches the destination successfully. The reason is that the robot will choose the new sub-target when the robot is close to the obstacle and goal simultaneously. This will change the attractive force's effect on the robot. With the help of the resultant force, the robot can reach the destination. The improved artificial potential field method successfully overcomes the problem that the robot is not able to reach the destination if the obstacle is too close to the goal.

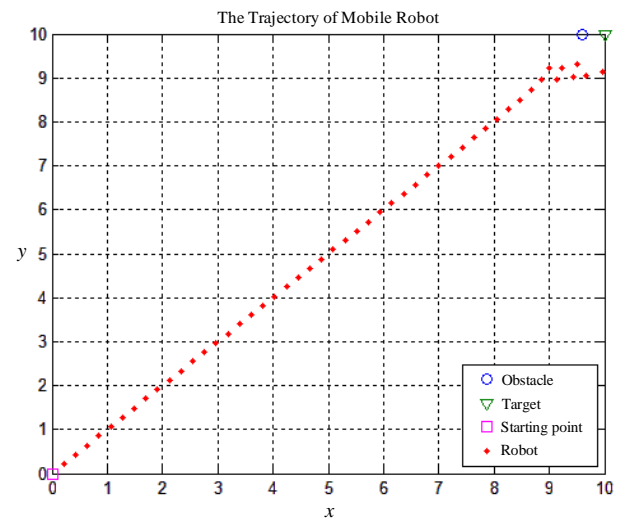

(a)

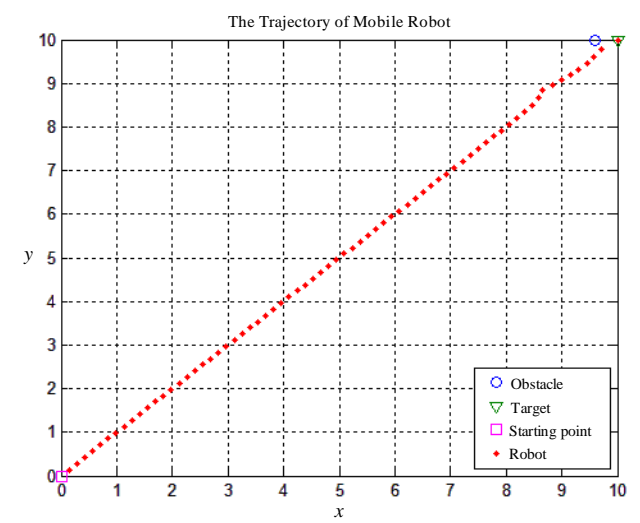

(b)

Fig. 6. Simulation comparison of the condition that the obstacle and goal are nearby: Traditional artificial potential field method (a); improved artificial potential field methods (b)

\section{Path planning experiment based on "Future Star" robot}

\subsection{Building the path planning experiment platform}

"Future Star" robot was selected to construct the physical experimental platform. The experiment used the path planning method and a part of the map was known. In the robot's program the experimental field, the initial position and goal's position were already defined. The position of the obstacle was recognized by the robot. The obstacle was a square box and the goal was an orange basketball. The robot could 
calculate the attractive force and move forward to the goal. The robot was always scanning the obstacles along its forwarding path. When the robot was close enough to an obstacle, the repulsive force of the obstacle made the robot bypass the obstacle. Due to the fact that the initial position and destination of the robot were known in advance, and only the position of the obstacle had to be detected by the sensors, the planned path was under the condition that a part of the map was known.

Fig. 7 shows the experimental environment. The $x$ axis was the direction along the robot which is $[0,400]$ (totally $4 \mathrm{~m}$ ). The right edge was the $y$ axis $[0,800]$ (totally $8 \mathrm{~m}$ ). The robot set out at coordinate $[150,0]$. The obstacle is located at $[200,150]$ and the destination is $[350,400]$.

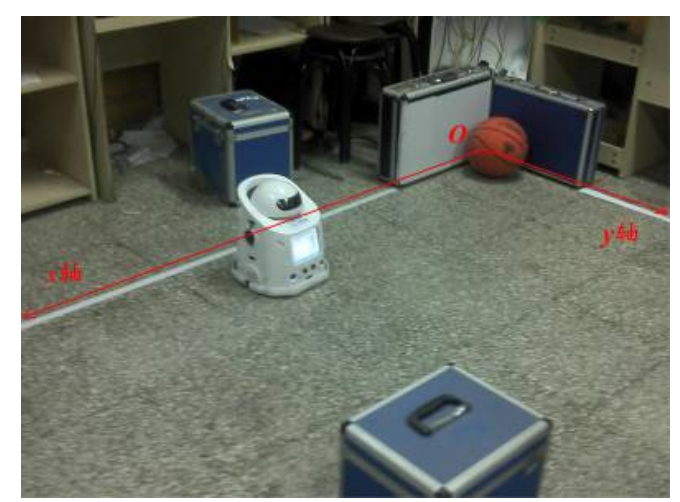

Fig. 7. Calibration of the coordinate

\subsection{Description and analysis of the experiment}

In Fig. 8a the robot set out at an initial position and prepared to move towards the goal. In Fig. 8b, the robot changed its direction because of the attractive force and was about to move to the destination. Fig. 8c showed that the robot had already aimed to the destination, though there was an obstacle, which was ahead of the robot.

Fig. 8d, e, $\mathrm{f}$ indicated that the ultra-sonic sensor, which was used to measure the distance detected a blue obstacle in front of the robot (as shown in Fig. 8d). The repulsive force affected the robot when the distance to the obstacle was too close (when the distance was less than $\rho_{\mathrm{O}}$ as Fig. 8d shows). The robot changed its direction to avoid the obstacle and moved to the goal at the minimal cost (shown in Fig. 8f). 


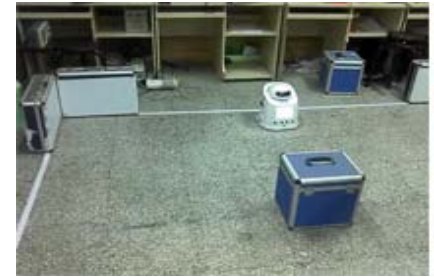

(a)

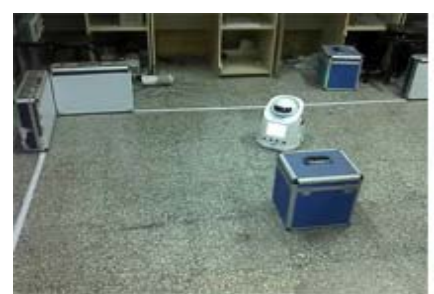

(d)

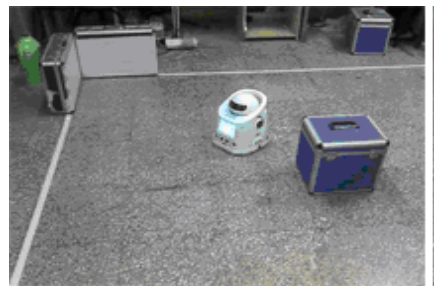

(g)

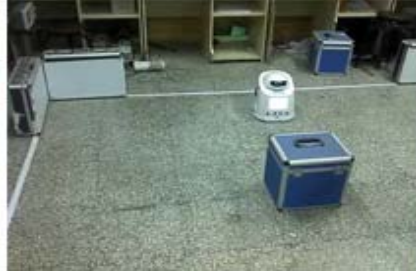

(b)

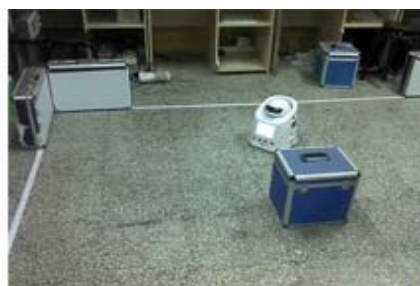

(e)

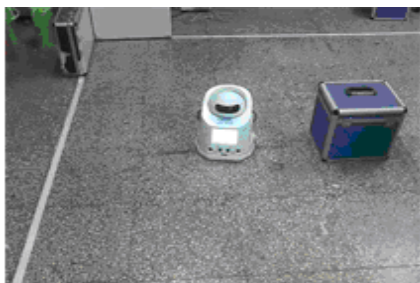

(h)

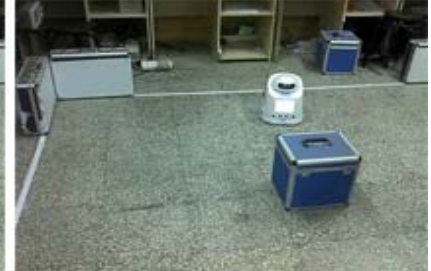

(c)

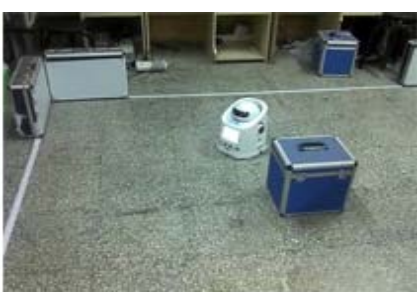

(f)

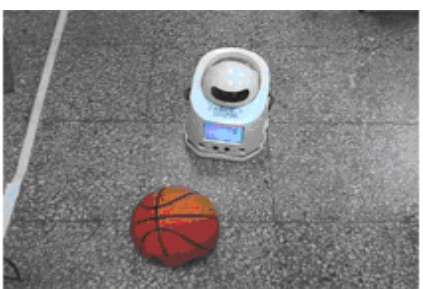

(i)

Fig. 8. Path planning with a single obstacle

In Fig. 8g the robot bypassed the obstacle successfully and adjusted its direction to move forward to the goal. In Fig. 8h the robot was on the side of the obstacle. Fig. 8i indicated that the robot was about to reach the destination successfully.

\section{Conclusion}

In this paper chaos optimization is introduced in the artificial potential field method and some problems, such as local minima and unreachable goal existing in the traditional artificial potential field method are solved. Comparison and analysis of the improved method proposed in this paper and the traditional artificial potential field method are performed by simulation. In addition, the improved method is also verified by building up a physical platform based on "Future Star" robot.

The simulation results show that the improved method proposed in this paper gets rid of the drawbacks of the traditional artificial potential field method depicted above. In addition, the planned path is smoother and easier to track. The success of the physical experiment further indicates the rationality and efficiency of the improved algorithm, which can be applied to mobile robot path planning. 
Acknowledgments: This work was partially supported by the Importation and Development of HighCaliber Talents Project of Beijing Municipal Institutions (No CIT\&TCD201404125), Youth Innovation Fund program of AGRS (2013YFL06) and Comprehensive Reform of Personnel Training (PXM2014_014224_000091).

\section{References}

1. R i m o n, E., D. E. K o d i t s c h e k. Exact Robot Navigation Using Artificial Potential Functions. - IEEE Trans. on Robotics and Automation, Vol. 8, 1992, pp. 529-551.

2. V a d a k k e p a t, P., K. C. T a n, W. M i n g-L i a n g. Evolutionary Artificial Potential Fields and Their Application in Real Time Robot Path Planning. - In: Proc. of 2000 Congress on Evolutionary Computation, 16-19 July 2000.

3. Q i x i n, C., H. Y a n w e n, Z. J in g l i a n g. An Evolutionary Artificial Potential Field Algorithm for Dynamic Path Planning of Mobile Robot. - In: Proc. of International Conference on Intelligent Robots and Systems(IEEE/RSJ), 9-15 October 2006.

4. K h a t i b, O. Real-Time Obstacle Avoidance for Manipulators and Mobile Robots. - In: Proc. of International Conference on Robotics and Automation, 25-28 March 1990.

5. Revello, T. E., R. M c C a r t n e y. A Cost Term in an Evolutionary Robotics Fitness Function. - In: Proc. of Congress on Evolutionary Computation, 2000.

6. Ge, S. S., Y. J. Cu i. New Potential Functions for Mobile Robot Path Planning. - Trans. on Robotics and Automation, Vol. 16, 2000, No 5, pp. 615-621.

7. Agirrebeitia, J., et al. A New APF Strategy for Path Planning in Environments with Obstacles. - Mechanism and Machine Theory, Vol. 40, 2005, pp. 645-658.

8. S h i, H., Z. Ch e n, C. S un. Application of Chaotic Optimization Algorithm to Problems with Motion Planning for Mobile Robots. - In: Proc. of 2003 IEEE International Conference on Robotics, Intelligent Systems and Signal Processing, October 2003.

9. Motl a gh, O. R. E., T. S. H o n g, N. I s m a i l. Development of a New Minimum Avoidance System for a Behavior-Based Mobile Robot. - Fuzzy Sets and Systems, Vol. 160, 2008, pp. 1929-1946.

10. L a, Y. L., S. H o n g, J. H u a n g. The New Environment Model Building Method of Penetration Mission Based on the Artificial Potential Field Approach. - In: Proc. of 2012 IEEE International Multi-Disciplinary Conference on Cognitive Methods in Situation Awareness and Decision Support, 2012.

11. Fathy, E., K. El-Metwally, A. R. Han a fy. Multi-Robot Tracking of Multiple Moving Targets Using Potential Field Approach. - In: Proc. of International Symposium on Innovations in Intelligent Systems and Applications, 2010.

12. L orenz, E. N. Deterministic Non-Periodic Flow. - Journal of the Atmospheric Sciences, Vol. 20, 1963, No 2, pp. 130-141.

13. Y a n g, D., G. L i, G. C h e n g. On the Efficiency of Chaos Optimization Algorithms for Global Optimization. - Chaos, Solitons and Fractals, Vol. 34, 2007, pp. 1366-1375.

14. B or e n s te in, J., Y. K or e n. Real-Time Obstacle Avoidance for Fast Mobile Robots. - IEEE Trans. Syst., Man, Cybern., Vol. 19, 1989, No 9, pp. 1179-1187.

15. Koren, Y., J. B orenstein. Potential Field Methods and Their Inherent Limitations for Mobile Robot Navigation. - In: Proc. of IEEE Conf. Robotics and Automation, Sacramento, 7-12 April 1991.

16. Khoa, T. Q. D., M. Naka gawa. Training Multilayer Neural Network by Global Chaos Optimization Algorithms. - In: Proc. of Int. Joint Conf. on Neural Networks (IJCNN'2007), August 2007.

17. S h i, H., Z. Che n, C. S u n. Path Planning Method for Robot Based on Chaotic Optimization Algorithm. - Robot, Vol. 27, 2005, No 2, pp. 153-157. 